\title{
Klienten und Kollegen
}

\section{Was engagementsuchende Wohlfahrtsverbände von genossenschaftlichen Prinzipien lernen können}

\author{
Peter-Georg Albrecht
}

\begin{abstract}
Das Ehrenamt gilt als eine der identitätsstiftenden Quellen der Freien Woblfahrtspflege. In der Praxis jedoch wird das freiwillige Engagement allerdings von Verantwortlichen und Fachkräften oft als zusätzliche Last empfunden. Genossenschaftliche Leitlinien wie Demokratieprinzip und Identitätsprinzip könnten eine neue Sichtweise eröffnen.
\end{abstract}

Alle Wohlfahrtsverbände rufen nach engagierten Bürgern. "Weil Menschen Menschen brauchen " gilt es, "generationsübergreifende Freiwilligendienste zu initiieren und zu institutionalisieren ", so eine Zielstellung des Diakonischen Werkes (Diakonisches Werk 2005, vgl. auch 2007). Auch die Arbeiterwohlfahrt versucht, durch neue Projekte wie die Initiative Ehrenamt "neue Zugänge des Engagements in den Verband zu eröffnen " (so die Bundestagsenquetekommission zur Zukunft des bürgerschaftlichen Engagements über die AWO 2002: 572, vgl. auch AWO 2007). Dem Deutschen Roten Kreuz geht es verbandspolitisch darum, »ehrenamtliche Mitwirkung zu stärken " (DRK 2007). Laut seiner strategischen Ziele will der Deutsche Caritasverband "ein effizienter und schlagkräftiger Verein «, sein, »mit dem sich seine Mitglieder identifizieren « und der »zivilgesellschaftliches Engagement stärker integriert « hat (Deutscher Caritasverband 2006). Freiwillig Engagierte sollen »maßgeblich die Zukunftsfähigkeit mitbestimmen «, so die ostdeutsche Volkssolidarität, mitgliederstärkste Organisation im Paritätischen Wohlfahrtsverband (Volkssolidarität 2002: Abs. 3).

Dr. Peter-Georg Albrecht lehrt an der Hochschule Magdeburg-Stendal im Fachbereich Sozial- und Gesundheitswesen. Internet http://www.hs-magdeburg.de
Bei genauerem Hinsehen fällt auf, dass zwischen diesen Zielstellungen und der Praxis der Engagementförderung eine große Lücke klafft. Allzu offensichtlich ist, dass der wohlfahrtsverbandliche Zug der marktbezogenen Ökonomisierung und der politischen Neupositionierung in eine andere Richtung rollt: Auf dieser Fahrt, die an Geschwindigkeit gar noch zuzunehmen zu scheint, sind freiwillig Engagierte eher irgendwie störend, sieht man sie doch in den oberen Verbandsetagen als Unprofessionelle an, deren Beiträge weder wirtschaftlich nutzbringend sind noch die politische Durchsetzungskraft stärken und die den Geschäftsführungen vor allem Zeit rauben und so das verbandliche Vorwärtskommen eher behindern.

Besonders deutlich wird die Wirklichkeit dieser informellen Werthaltung, sieht man auf die Soziale Arbeit an der Basis und die dort stattfinden Prozesse der Professionalisierung. Auch in der Engagementförderung der Sozialen Arbeit wird hinter vorgehaltener Hand - auf den Unterschied gepocht: hier der Professionelle, dort der Laie, hier der gut Ausgebildete, dort der "aus dem Bauch heraus" Handelnde, hier der Kontinuität gewährleistende Verantwortungsträger, dort der Unberechenbare. Freiwillig Engagierte sind neues Klientel der Sozialen Arbeit, das "nun auch noch " bedient werden soll, so wird es vielfach empfunden, wurden freiwillig Engagierte doch bisher vor allem als ambivalente Partner der »wirklich Bedürftigen " angesehen, nicht aber als direkte Partner der Sozialarbeiter.

Diese Haltung in den Chefetagen wie auch an der Basis ist bedauerlich, birgt doch gerade die gegenwärtige Bewegung in der Engagiertförderung die Chance, die Mitgliedersituation von Wohlfahrtsverbänden zu verbessern.

Damit dies gelingen kann, gilt es zunächst, sich die durch den Staatskorporatismus und die neue Wirtschaftlichkeit eingeschlichenen Hierarchisierungen und die vieles ausblendenden Erlösbestrebungen einzugestehen, die die Schere zwischen oben und unten, zwischen der Leitung und den Angestellten, zwischen politischen und wirtschaftlichen Geschäftsführungen und Sozialarbeitern auseinandergetrieben hat und stets auch die Gräben zwischen den Verbänden und ihrem Klientel vertieft.

Das Konzept der Sozialgenossenschaftlichkeit (vgl. Flieger 2003) empfiehlt andersartige inner- und außerorganisatorische Strukturen, die Wohlfahrtsverbänden helfen können, engagiertenfreundlicher zu werden. Sozialgenossenschaften basieren auf der Gemeinschaft und dem Gemeinschaftsgeist ihrer Mitglieder, auf der Erfahrung, dass kooperatives Handeln lohnt und dem Vertrauen, dass die Mitglieder in guten wie auch in schlechten Tagen füreinander einstehen.

Am deutlichsten wird dieses Solidaritätsprinzip wohl dadurch, dass alle Mitglieder einer Sozialgenossenschaft gleichermaßen an grundsätzlichen Entscheidungen beteiligt sind und die Gestaltung der täglichen Arbeit miteinander abstimmen (Demokratieprinzip). In allen Genossenschaften gilt, dass Anbieter gleichzeitig Nachfrager, in der Wohnungsbaugenossenschaft Vermieter ebenso Mieter, in der Produktivgenossenschaft Besitzer auch Beschäftigte und Produktnutzer, in der Konsumgenossenschaft Verkäufer auch Käufer und Verbraucher und in der Sozialgenossenschaft Träger der Wohlfahrtsproduktion auch die Nutzer derselben sind (Identitätsprinzip).

Zentral ist das Förderprinzip, das darauf verweist, dass durch die genannte Auflösung der Rollen und den ihnen innewohnenden hierarchie- und abgrenzungsfördernden Kräften alle Mitglieder vom kooperativen Handeln profitieren. Darüber hinaus entsteht, das muss an dieser Stelle erwähnt werden, vielfach auch für Nichtmitglieder und das Gemeinwesen, in dem Sozialgenossenschaften agieren, ein Nutzen. 
Das Konzept der Sozialgenossenschaftlichkeit kann Wohlfahrtsverbänden zeigen, dass es diese Prinzipien braucht, um Zufriedenheit, Kooperationsbereitschaft und Produktivität bei Mitarbeitern herstellen zu helfen, denen ihr Mitgliederstatus bewusst wird. Vor allem aber verweist es darauf, wie es möglich ist, die innerorganisatorischen Distanzen zwischen Leitung und Angestellten und die die Außenbeziehungen bestimmenden Gräben zwischen »den Kollegen « im Verband und dem Klientel »dort draußen « zu überwinden sind.

Wirklich engagiertenfreundliche Wohlfahrtsverbände sind stets bemüht, aus Klienten Kooperationspartner und schließlich Kollegen zu machen. Das genossenschaftliche Solidaritätsprinzip empfiehlt, dass sich die Mitarbeiter Wohlfahrtsverbände wieder neu ihrer »Dienstgemeinschaft «, wie es die konfessionellen Verbände nennen, bewusst werden.

Das Demokratieprinzip legt nahe, stets möglichst viele Menschen in die alltäglichen Reflexionen und Planungsprozesse einzubeziehen und vor allem Grundsatzentscheidungen breit zu diskutieren. Besonders das Identitätsprinzip empfiehlt, die klassischen Rollenverteilungen und Hierarchien aufzulösen und die entsprechenden Gräben zu überwinden. Dass man aus sozialem Engagement, sei es im Hauptamt wie im Ehrenamt, sei es als Geschäftsführer oder Sozialarbeiter, guten Gewissens auch einen persönlichen "Gewinn « ziehen kann, das erlaubt das genossenschaftliche Förderprinzip. Natürlich muss all das Erwähnte unter den wohlfahrtsverbandlichen Zielen der Gemeinnützigkeit, der Mildtätigkeit und der Sozialen Arbeit zusammenkommen (auch wenn manche, die Genossenschaften bewerten, meinen, das sei schwer möglich).

Doch ein solcher Spagat muss sein, die entsprechenden Ambivalenzen sind nicht zu umgehen, kommt doch Engagiertenfreundlichkeit vor Engagementförderung, gilt es, in den Geschäftsführungen die "Klotz-am-Bein-Brille « und in der Sozialen Arbeit die »Klientenbrille « gegenüber freiwillig Engagierten abzunehmen.

\section{Literatur}

Altvater, E./Sekler, N. (2006): Solidarische Ökonomie. Hamburg, VSA Verlag.

Elsen, S. (2007): Die Ökonomie des Gemeinwesens. Sozialpolitik und Soziale Arbeit im Kontext von gesellschaftlicher Wertschöpfung und -verteilung. Weinheim, Juventa Verlag.

Keupp, H. (2007): Plädoyer für eine zivilgesellschaftliche Neuerfindung Sozialer Arbeit. In: TUP - Theorie und Praxis der Sozialen Arbeit. 3/2007. S. 11-18.

Flieger, B. (2003): Sozialgenossenschaften als Perspektive für den sozialen Sektor in Deutschland. In: Flieger, B. (Hg.): Sozialgenossenschaften. Wege zu mehr Beschäftigung, bürgerschaftlichem Engagement und Arbeitsformen der Zukunft. Neu-Ulm, Bundesverein zur Förderung des Genossenschaftsgedankens. S. 11-35.

AWO Bundesverband (2007): Grundsätze und Eckpunkte zur Verbandsentwicklung der Arbeiterwohlfahrt. Magdeburg, Beschluss der ArbeiterwohlfahrtBundeskonferenz am 22./23.06.2007.

Bundestagsenquetekommission "Zukunft des Bürgerschaftlichen Engagements « (2002): Bürgerschaftliches Engagement: Auf dem Weg in eine zukunftsfähige Bürgergesellschaft. Bericht. Opladen, Leske \& Budrich.

Deutscher Caritasverband (2006): Strategische Ziele des Vorstands des Deutschen Caritasverbandes für die Jahre 2007 bis 2011. In: Neue Caritas 4/2007. S. 28-29. Freiburg i.Br. und Berlin, Vorstand und Caritasrat des Deutschen Caritasverbandes.

Diakonisches Werk der EKD (2007): Versöhnt und evangelisch profiliert - eine erste strategische Skizze. Münster, Diakonische Konferenz vom 16. bis 18.10.2007.

Diakonisches Werk der EKD (2005): Pressemitteilung. In: www.diakonie.de/ de/ html/ presse/ 3037_3483.html. Eingesehen am 05.03.2008.

DRK Bundesverband (2007): Jahrbuch 2006/2007. Berlin, Präsidium des Deutschen Roten Kreuzes.

Volkssolidarität Bundesverband (2002): Zukunftsorientierung der Volkssolidarität. Kurzfassung. Berlin, Beschluss der Bundesdelegiertenversammlung am 23.03.2002.

Möglicherweise bedarf es für eine "zivilgesellschaftliche Neuerfindung Sozialer Arbeit « (so Keupp 2007) und zur Überwindung der Unterscheidung in oben und unten und des Angebot- und Nachfragegegenübers einer gemeinsamen Vision. Diese kann, vor dem Hintergrund genossenschaftlichen Denkens, nur die Vision einer solidarischen Ökonomie und einer sozialen Politik sein, sowohl in wohlfahrtsverbandlicher Einzelperspektive als auch aus Sicht des Gemeinwesens, dem originären Ort - wie es Susanne Elsen (2006) formuliert - "gesellschaftlicher Wertschöpfung und -verteilung «.

Neuerscheinung November 2011

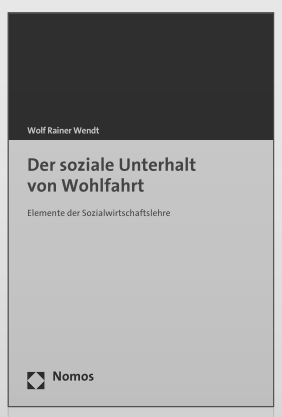

\section{Der soziale Unterhalt von Wohlfahrt}

Elemente der

Sozialwirtschaftslehre

Von Wolf Rainer Wendt

2011, 178 S., brosch., 39,-€

ISBN 978-3-8329-7000-0

www.nomos-shop.de/14092

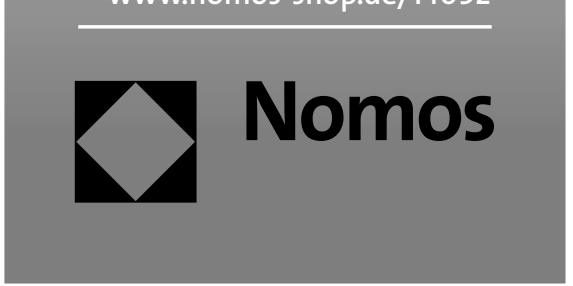

